\title{
A New Version of Modified Camel Algorithm for Engineering Applications
}

\author{
Ramzy S. Ali ${ }^{1}$, Jawad R. Mahmood ${ }^{2}$, Hussein M. Badr ${ }^{3}$ \\ \{ramzy.ali@uobasrah.edu.iq ${ }^{1}$, Jawad.mahmood@uobasrah.edu.iq ${ }^{2}$, \\ cse.61104@uotechnology.edu.iq $\left.{ }^{3}\right\}$ \\ Electrical Engineering Department, College of Engineering, University of Basrah, Iraq ${ }^{1,2}$ \\ Training and Energy Research Office, Ministry of Electricity, Iraq, Baghdad ${ }^{3}$
}

\begin{abstract}
The new modified camel algorithm (NMCA) is presented as a novel optimization method for tackling optimization problems in this research. The modified camel algorithm (MCA) and other metaheuristic algorithms are not the same as NMCA. Where it offers a fresh perspective on global optimization. The suggested method is validated using power distribution system challenges (engineering problems) that are frequently encountered in the optimization field. The IEEE 69- and 33-bus systems were used in the simulations. The NMCA algorithm was able to find the best answers in a variety of test circumstances. The findings of the NMCA are compared to those of the MCA and other well-known optimization techniques. The findings suggest that the NMCA is capable of addressing optimization problems effectively.
\end{abstract}

Keywords: New modified camel algorithm; distributed generation; solar photovoltaic; congestion problem; power loss; voltage profile.

\section{Introduction}

The use of metaheuristic algorithms expands considerably as the spatial and temporal complexity of power resource management problems grows. To tackle various challenges in power resources and engineering difficulties in general, many algorithms are presented and utilised. Solar photovoltaic (PV) power is one of the most important power sources. In distribution grids, the photovoltaic distribution generator (PVDG) is one of the most promising renewable power sources. PVDG generation capacity has expanded significantly in recent years [1-2] because to its flexibility in scale application and pollution-free nature.

According to the literature review, optimization methods are commonly employed to solve complicated power system challenges (optimization problems). In comparison to traditional methods based on mathematical programming, metaheuristic algorithms are generally more powerful [3]. The researcher in [4] investigates the topic of network reconfiguration in the presence of distributed generation (DG) by employing a Harmony Search Algorithm (HS) to concurrently reconfigure and find the best places for DG unit installation in a distribution grid. Amin [5] presented a hybrid configuration of particle swarm optimization (PSO) with ant colony optimization (ACO) algorithm called hybrid PSO-ACO algorithm to solve the distribution network minimizing power losses problem and improving the voltage profile problem. The major points in the modified camel traveling algorithm are the diversification and intensification 
[6-7]. The diversification phase ensures that the MCA efficiently traverses the search space, while the intensification phase sorts through the current best solutions and chooses the best candidates. MCA was created in order to solve engineering problems more quickly and with more reliable solutions [6]. For global optimization, a new modified camel algorithm (NMCA) is introduced in this study. Congestion difficulties and distribution network losses are used to test the NMCA's performance and efficiency. The results show that NMCA may be used to solve distribution network optimization problems. In addition, the NMCA can outperform existing optimization methods..

The following is a breakdown of the paper's structure: Section 2 introduces the MCA and NMCA, as well as the suggested NMCA's properties and algorithm formulation. Section 3 contains the problem formulation and constraint. The NMCA algorithm's usefulness for congestion, voltage profile power losses, and other problems is discussed in Section 4. The suggested algorithm's performance is evaluated in section 5 using congestion problems, voltage profiles, and power losses in distribution networks. The suggested NMCA algorithm's convergence mobility is discussed in Section 6. Finally, in Section 7, there are concluding observations and recommendations for future research.

\section{The Camel Travelling Algorithm}

The Camel Travelling Algorithm (CA) is a swarm intelligence-based technique proposed by Ramzy and Mohammed in 2016 [8]. CA is inspired by the traveling behaviour of camels during foraging. The $\mathrm{CA}$ algorithm has a complex structure that negatively affects the execution speed and memory size, where the CA consists of multi-loop nesting and several parameters selection. In 2019 Ramzy introduce the modified Camel traveling algorithm (MCA) with a simple structure, which significantly improve its convergence and computation speed [6]. The MCA is presented to solve engineering optimization problems. In this section, a new version of the MCA algorithm (New-MCA) is presented. The NMCA uses to find the optimum configuration (size, position, and number) of the PV units in the distribution grid to solve the congestion problem, improve voltage profile and reduce power losses. NMCA works in the same manner as MCA, with the following modification:

1. Modification of the mutation step.

2. Modification of the location equation.

3. Addition of the velocity equation.

4. Addition of the velocity limits.

5. Addition of the location limits.

Simulation of camel visibility in the MCA algorithm is employed in the same manner as the mutation simulation in other algorithms like the GA algorithm. There are two situations for updating each camel's location in the MCA algorithm. The update function is a switch from the main location equation to the second location equation when the camel visibility $\mathrm{v}$ exceeds a certain visibility threshold. The goal of modified mutation in NMCAs is to add variety to the camel sites. Mutation operators are employed to avoid local minima by avoiding camel sites from getting too identical to one another, which slows or even stops convergence to the global optimum. 
The visibility of the camel and the simple location equation in MCA, inefficient for solving congestion problems and other distribution problems due to the following reasons:

1. The mutation step in the food searching equation must not take place in a position taken close to the food source because this affects the solution convergence.

2. The updated locations of camels might not generate a feasible solution and rounding off the new food source becomes essential.

3. MCA algorithm does not have limits applied on location equation where this affects the solution.

In NMCA, the modified mutation process was included to keep a certain level of heuristic knowledge. In the original MCA, the camels will look for a new food source in their recollection of a nearby site. In NMCA, the camels will look for a new food supply in a nearby place that they remember, and they will retain certain knowledge from the stored favourable results. The optimization algorithm will quickly converge as a result of this method. A camel develops a candidate food position by probabilistically modifying the camel location in her memory for finding a new food source. It is assumed that there are $\mathrm{N}$ camels (Camel Caravan) going through a (D) dimensional camel environment for the implementation of the new modified camel algorithm. Where $x^{i, i t r}$ denotes the location of the camel I at the time of iteration (itr):

$x^{i, i t r}=\left\{x_{1}^{i, i t r}, \quad x_{2}^{i, i t r}, \quad \ldots, \quad x_{D}^{i, i t r}\right\}$

and

$i=1,2, \ldots, N$.

itr: Iteration, $i t r=1,2, \ldots, i t r_{\max }$.

At the beginning (itr $=0$ ), where the camels are spread in the desert and looking randomly for the water supply and food as demonstrated in the formula below:

$\gamma=\left(x_{\max }-x_{\min }\right)$

$x_{d}^{i t r}=\gamma R+x_{\min }$

Where

$R \in[0,1]$

$d=1,2, \ldots, D$.

$x_{d}^{i t r}:$ Location of a camel.

$x_{\min }$ : Minimum location of a camel.

$x_{\max }$ : Maximum location of a camel.

$\gamma$ : Rang of camel location

The temperature (T) and effect of the temperature on the camel endurance $(E)$ of camel $i$ at the time iteration as shown below:

$T_{d}^{i t r}=\left(T_{\max }-T_{\min }\right)$ Rand $+T_{\min }$

$E_{d}^{i t r}=\frac{\left(T_{d}^{i t r}-T_{\min }\right)}{\left(T_{\max }-T_{\min }\right)}$ 
Where

$T_{\text {min }}$ : Min amount of temperature

$T_{\text {max }}$ : Max amount of temperature

$T_{d}^{i t r}:$ Temperature

$E_{d}^{i t r}:$ Endurance

The camel velocity and velocity limits terms can be mathematically expressed by:

$v_{\max }=0.25 \gamma$

$v_{\min }=-v_{\max }$

$v_{d}^{i t r}=\Theta v_{d}^{i t r-1}+\left(x_{d}^{b e s t}-x_{d}^{i, i t r-1}\right) E_{d}^{i, i t r} * c$

Where

$v_{\text {min }}:$ Minimum velocity of a camel.

$v_{\max }$ : Maximum velocity of a camel.

$v_{d}^{i t r}$ : Velocity of a camel.

Note: $c \in[1,3]$ and $\theta \in[0,1]$ are constants.

Finally, the main location equation is:

$x_{d}^{i t r}=x_{d}^{i t r-1}+v_{d}^{i t r}$

Algorithm: New Modified CA

Begin

Step 1: Initialization: Set the temperature range and the location range Tmin and Tmax; set the camel caravan size and the dimensions; set the visibility threshold; set std. threshold; set $\mathrm{c}$ and $\theta$ values; initialize the location of each camel from Eq. (3).

Step 2: Subject the locations to a certain fitness function; determine the current best location; randomly assign visibility (v) for each camel.

Step 3: While $\left(\boldsymbol{i t r}<\boldsymbol{i t r}_{\text {max }}\right)$ do

for $\mathrm{i}=1$ : Camel Caravan size

Compute the temperature T from Eq. (4)

Compute the endurance E from Eq. (5)

Compute the Std. of Best Costs.

Update the camel velocity from Eq. (8)

Apply velocity limits

If $v<$ visibility threshold and Std. $<$ Std. threshold then else

Update the camel location from Eq. (3)

Update the camel location from Eq. (9) end If 


Apply location limits
end for

\section{Problem Formulation}

In today's modern power systems with large loads and renewable sources, including solar power, network security limits, such as thermal, angular stability, or voltage stability are violated. When renewable energy penetration is high, it can have a negative impact on power flow in the system and cause congestion in transmission and distribution systems. In electricity systems, a congestion problem occurs when demand for transmission capacity exceeds transmission grid capabilities. While congestion reduction will become increasingly crucial for this reason [9]. This research focuses on the network's congestion problem at the distribution level.

\subsection{Objective Function}

Total active power losses are one of the four key components of the multi-objective function; the L $\infty$ to handle the voltage profile, congestion lines in the distribution network, and PVDG which are installed at the distribution level.

\subsubsection{Total Active Power Loss}

In the optimal operation of a working power system, total active power loss (TPL) can be considered, the major factor of economic performance, where this issue is the main objective in the optimal power system. The mathematical expression of total active power loss is given equation (10) $[2,9]$ :

$T_{1}=T P L=\sum_{n=1}^{N_{b r}} I_{n}^{2} R_{n}$

$N_{b r}:$ several branches in the system.

$\mathrm{I}_{\mathrm{n}}$ : the current magnitude of the $\mathrm{n}^{\text {th }}$ branch.

$\mathrm{R}_{\mathrm{n}}$ : The resistance of the $\mathrm{n}^{\text {th }}$ branch.

The TPL will be from zero to $10^{\text {th }}$ of MW. 


\subsubsection{The Congestion Lines in the Distribution System}

One of the factors that prevent the integration of PVDG power in a distribution system is the congestion of a distribution grid due to the limited availability of distribution grid capacity. Therefore, the selection of the maximum congestion line in the distribution grid is the major factor in the objective function to solve the congestion problem in this study. The selection of the maximum congestion line is performed by employing the contingency ranking. The performance index is the main factor used for ranking the system. The circuit current-based index is used to represent the line overloads as in equation (11):

$J_{C}=\sum_{j} W_{j}\left(\frac{I_{j}}{I_{N, j}}\right)^{2 n}$

$j=1,2, \ldots, m j$.

Where,

$j:$ number of lines.

$W_{j}$ : weighting factor, $0<W_{j}<1$.

$I_{N, j}:$ thermal limit of the current line.

$I_{j}:$ actual current through circuit $j$.

$n:$ the exponent factor.

The term of maximum congestion line in objective function depends on the relation between the magnitude of actual current through the circuit and current-based thermal limit of the line as in equation (12) [10]:

$T_{2}=\max \left(\frac{I_{j}}{I_{N, j}}\right) * 100$

\subsubsection{PVDG Injected Power}

The PVDG is available in sizes from several kilowatts to tens of megawatts and is connected to the power system at the substation or distribution levels. To find optimal PVDG with minimum total power injected in distribution level, equation (13) is used in multi-objective function as[11]:

$T_{3}=\sum_{i=1}^{N_{P}} P_{P}, i$

Where,

$N_{P}$ : The number of the PVDG in the Distribution system.

$P_{P}:$ The power of the $\mathrm{g}^{\text {th }}$ PVDG. 


\subsubsection{The $L_{\infty}$ Technique}

The injection of PVDG in the radial distribution system has many benefits to the system. But it represents an influencing factor on the voltage rise in the system. The voltage rise is the bottleneck of PVDG in the radial distribution system. Therefore, many previous studies attempted to address the question of how much PVDG can be penetrated in a distribution grid without risking overvoltage. In this study, the $L_{\infty}$ the technique is proposed to improve the voltage profile, with an optimal algorithm to select the optimal size and placement of PVDG.

The $\infty$-norm: As $p \rightarrow \infty$, the $L_{p}$ norm tends to the so-called $\infty$-norm, or $L_{\infty}$ norm, which defines the $L_{-} \infty$ norm as the supremum (least upper bound) of the absolute value as in equation (14) [12]:

$\|x\|_{\infty}=\sup _{t}|x(t)|$

For the vector $V=\left\{v(1), \ldots, \quad v\left(N_{b u s}\right)\right\}^{T}$, with, $i=1,2, \ldots, N_{b u s^{*}}$, where the $N_{b u s}$ is the number of buses in the distribution system. $V$ is the measured voltage of the distribution system and, $v(i)$, is the measured voltage at the bus $(i)$ in the distribution system. To find the supremum of the vector $V$ :

$\|V\|_{\infty}=\sup _{i}|v(i)|$

$v_{p}(i)=(v(i)-1) * 100$

$V_{P}=\left\{v_{p}(1), \quad \ldots, \quad v_{p}\left(N_{\text {bus }}\right)\right\}^{T}$

Now, the $L_{\infty}$ norm will be applied to $V_{p}$ to find the supremum of the vector $V_{p}$, where the $V_{p}$ vector can represent the values of voltage change at each bus in the distribution system

$T_{4}=\left\|V_{p}\right\|_{\infty}=\sup _{i}\left|v_{p}(i)\right|$

The multi-objective function of the PVDG in the grid is considered as follows:

$T=\left(K_{1} T_{1}\right)+\left(K_{2} T_{2}\right)+\left(K_{3} T_{3}\right)+\left(K_{4} T_{4}\right)$

Here, $T$ is the fitness function which is required to be minimized to reduce congestion and get minimum losses with $K_{1}, K_{2}, K_{3}$ and $K_{4}$ are represent the penalty factors.

\subsection{Constraints}

The objective function in equation (19) is subjected to a set of constraints, as shown below:

\subsubsection{Balance Constraints of the Applied Power}

The flow of current via impedances of electrified conductors causes power losses, which can be computed using equation (10). The power balance restriction is expressed as [2]: 
$\sum_{i=1}^{N_{l}} P_{l}, i-P_{\text {Grid }}-\sum_{i=1}^{N_{P}} P_{P}, i+\sum_{n=1}^{N_{b r}} P_{\text {loss }}, n=0$

\subsubsection{The Voltage and Current Limits}

The distribution grid is modelled as buses connected by a set of transmission lines with PVDGs connect to the grid. Bus 1 is the slack bus and has a constant voltage magnitude and phase, and the buses $(i=2$, Nbus $)$ have variable voltages within allowable limits to ensure the required quality of service of the system. The lower and upper bounds for the voltage are 0.95 to $1.05 \mathrm{pu}$ in distribution systems [13].

$V_{\min } \leq\left|V_{i}\right| \leq V_{\max }$

$V_{i}$ : magnitude of the variable voltage.

$V_{\text {min }}:$ min voltage limits.

$V_{\text {max }}$ : max voltage limits.

The line current limitation in the transmission line between buses ( $i$ and $j$ ) is imposed as in equation $(22)[2,13]$ :

$I_{i j} \leq I_{i j}^{\max }$

\subsubsection{The PVDG Units' Capacity Limits}

The acceptable voltage limits in the distribution network will be violated due to the further addition of PVDGs, which cause an increase in network losses due to the reverse power flow. Thus, the hosting capacity of PVDG of the distribution network is limited by overvoltage. The power of each PVDG unit is select within a specific range:

$P_{P}^{\min } \leq P_{P} \leq P_{P}^{\max }$

Also, the sum of the capacity of all PVDG units should not be exceeded the load demand in the distribution grid as represented in equation (24):

$\sum_{i=1}^{N_{P}} P_{P}, i \leq \sum_{i=1}^{N_{l}} P_{l}, i$

\section{NMCA Optimization Algorithm for PVDG Units}

PVDG planning is one of the most essential concerns with considerable economic and technical implications. With the rapid development in the number of PVDG units connected to the power grid, multiple methods for solving optimization issues with various objective functions and constraints have been developed. An extra power source can be introduced to the grid by strategically placing and sizing PVDGs. This will help to improve the system and alleviate congestion. In this section, NMCA has been used to determine the appropriate location and size of PVDG units in order to alleviate congestion and reduce overall power loss. The following is a detailed description of the entire process of applying the NMCA algorithm for optimal PVDG unit allocation in a radial distribution power system: 
Step 1: Initialization:

Set the temperature range $T_{\min }$ and $T_{\min }$ and the location range set the camel caravan size and the dimensions, set the Visibility threshold, set Std. threshold set $c$ and $\theta$ values, initialize the location of each camel.

Upload system data, that includes bus and line data of the radial distribution system.

Define the constraints of the problem.

Step 2: Subject the locations to a certain fitness function, determine the current best location, and randomly assign visibility ( $v$ ) for each camel.

To calculate the fitness function:

Subject the solution (PV location and size) to the Distribution system.

Run power flow to obtain all variables.

Run contingency analysis.

Calculate $\mathbf{L} \infty$.

Calculate total power loss, then calculate fitness function (multi-objective function) by using Equation (19).

For r=1: Run

Step 3:

While (iter < itermax) do

For i=1: Camel Caravan size

Compute the temperature $T$.

Compute the endurance $\mathrm{E}$.

Compute the Std. of Best Costs.

Update the camel velocity.

Apply Velocity Limits

If $v<$ visibility threshold and Std. < Std. threshold then

Else

Update the camel location from Eq. (3)

Update the camel location from Eq. (9)

End If

Apply location limits

End for

Subject the new locations to the fitness function

Calculate the fitness function

If the new best location is better than the older one

The new best is the global best

End If

Update the Std. counter for best costs

Assign new visibility for each camel

Step 4: End While

Step 5: If the problem limits are satisfied then

Output the best solution

Else

Rule out the solution

End If

Step 6: If the new best solution is better than the older one.

Then the new best is the global best

End If

End for

End 


\section{The Radial Distribution System as a Testbed for the Proposed Algorithm}

The in this section, different case studies are conducted to evaluate the overall performance of the proposed strategy. Two IEEE test systems are considered here:

1. Scenario 1: normal load-case of 33-bus with PVDG.

2. Scenario 2: heavy load case of 33-bus with PVDG.

3. Scenario 3: normal load-case of 69-bus with PVDG.

4. Scenario 4: heavy load case of 69-bus with PVDG.

\subsection{Case Study (1)}

The proposed strategy is applied to IEEE 33 bus structure system. Here, the problem is to find an optimal configuration (the number, locations, and sizes) of PVDGs. This system has been modified such that the current load is 3.7 MW active power and 3.53 MVAR reactive power, whereas the original system with 3.7 MW active power and 2.3 MVAR reactive power [14]. the modification's purpose is to create a suitable environment for congestion problems and voltage droop problems. The power calculation has been done assuming Sbase $=100 \mathrm{MVA}$ and Vbase $=12.66 \mathrm{kV}$. Figs 1 and 2 reflect the system behaviour with and without PVDGs for normal loading conditions. Figures (1) show the system busses current with the presence and absence of PVDGs in addition with line limits. From the figure one can conclude the flowing points:

1- Reduction of lines current. Such that all the current droop below limits values.

2- Reduction of current has a significant effect on reduced power loss and relief the congestion in the system.

Fig 2 shows the voltage profile of the system under consideration with and without PVDGs installation. From the obtained result's one can see when there are no PVDGs the voltage profile suffers from noticeable voltage droop special in buses (16-17-18), where voltage droop to ( $0.8658 \mathrm{pu})$. but on the application of PVDGs, the system profile improved efficiently, and the voltage profile tolerated between (1 and 0.9901) pu.

\subsection{Case Study (2)}

In this case, the system has been tested for heavy loading represented by $5.1401 \mathrm{MW}$ active power and 4.942MVAR reactive power. also, the power calculation has been done assuming Sbase $=100 \mathrm{MVA}$ and Vbase $=12.66 \mathrm{Kv}$. From Fig 3 the following points are clear:

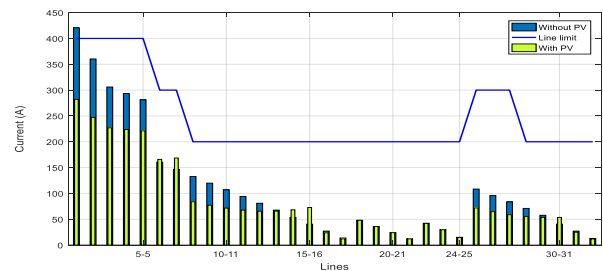

Fig. 1. Lines current without and with PVDG 33-bus system with a normal load

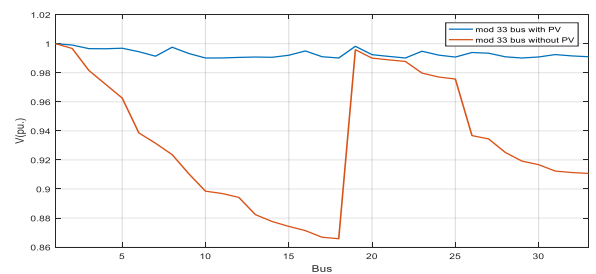

Fig. 2. Volt profile without and with PVDG units for the same bus of Fig. 1 
1. When there is no PVDGs power the system loading for lines (1-2,2-3, 3-4, 4-5, and 56 ) exceding their thermal limits and the system cannot deal with such loading specific in these buses.

2. On the application of PVDGs the bus's current droop below their thermal limits and there (inline (1-2) the current before installing PVDGs is (611A) and after installed PVDGs is (399A).

From Fig 4 the following points are clear:

1. When there are no PVDGs the system suffers from a high deviation in voltage profile where it reaches $(0.8003 \mathrm{pu})$ and the accepted limit in distribution grid is (1.05 to 0.95$)$ pu.

2. The PVDGs Improve the voltage profile when installed, where the deviation in the voltage profile reduces from $(0.8003 \mathrm{pu})$ to $(0.9861 \mathrm{pu})$.

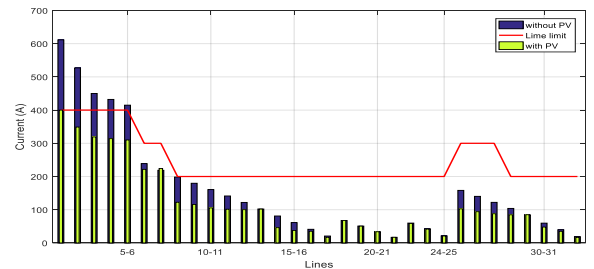

Fig. 3. Lines current with and without PVDG units, for a 33-bus system with a heavy load.

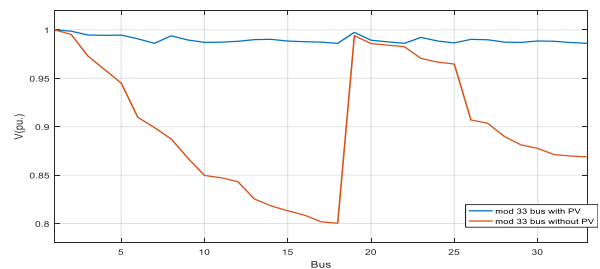

Fig. 4. Volt profile with and without PVDG units For the same bus of Fig. 3

\subsection{Case Study (3)}

The proposed strategy has been applied to IEEE 69 bus structure system. In this case, the system has been tested for normal loading represented by 3.8 MW active power and 2.7 MVAR reactive power [15]. also, the power calculation has been done assuming Sbase $=100 \mathrm{MVA}$ and Vbase $=12.66 \mathrm{Kv}$ Figure(5) and figure (6) demonstrate the results for applied PVDGs in IEEE 69 system. From figure (5), the following points are clear:

1. On the application of PVDGs the buses current droop below their thermal limits and there (inline (1-2) the current before installing PVDGs is (387.18 A) and after installed PVDGs is $(216.1 \mathrm{~A})$

2. The result of the reduction of current is power loss reduction and relief of the congestion in the system.

From the figures (6) the following points are clear:

1. When there are no PVDGs the system suffers from a high deviation in voltage profile where it reaches ( $0.9092 \mathrm{pu})$.

2. The voltage profile Improved when PVDGs has installed, where the deviation in the voltage profile reduces from ( $0.9092 \mathrm{pu})$ to $(0.9966 \mathrm{pu})$.

\subsection{Case Study (4)}


In this case, the system has been tested for heavy loading (1.6 from normal) represented by 6.082224MW active power and 4.30976 MVAR reactive power. also, the power calculation has been done assuming Vbase $=12.66 \mathrm{~K}$, and Sbase $=100$ MVA. Fig. 7 and Fig. 8 demonstrate the results for applied PVDGs in IEEE 69 system. From Figure 7, the following points are clear:

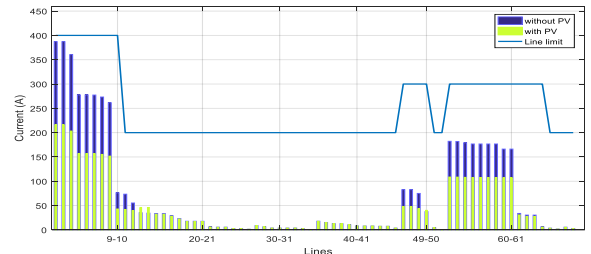

Fig. 5. Lines current without and with PVDG for 9bus system with a normal load

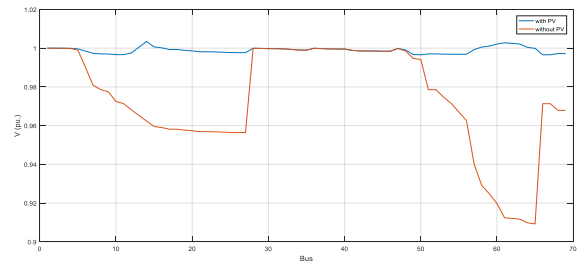

Fig. 6. Volt profile with and without PVDG units for the same bus of Figure 5.

1- When there is no PVDGs power the system loading for lines (1-2 , 2-3, 3-4 , 4-5, 5-6, $6-7,7-8,8-9,51-52,9-53,53-54,54-55,55-56,56-57,57-58$ and 58-59) exceeding there thermal limits.

2- On the application of PVDGs the buses current droop below their thermal limits and there (inline (1-2) the current before installing PVDGs is (644.3694 A) and after installed PVDGs is (348.5021 A).

From the Fig 8 the following points are clear:

1. When there are no PVDGs the system suffers from a high deviation in voltage profile where it reaches ( $0.8445 \mathrm{pu})$.

2. The PVDGs Improve the voltage profile when installed, where the deviation in the voltage profile reduces from ( $0.8445 \mathrm{pu})$ to $(0.9947 \mathrm{pu})$.

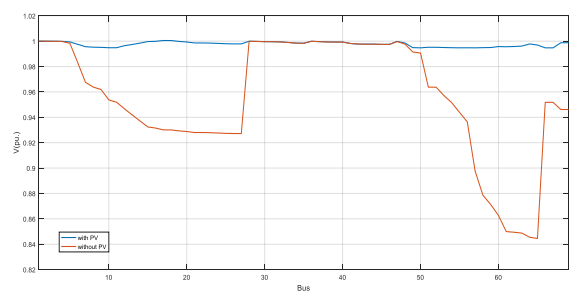

Fig. 7. Lines current with and without PVDG for a 69-bus system with a heavy load

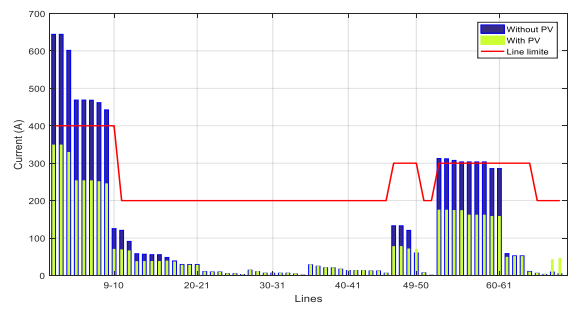

Fig. 8. Volt profile with and without PVDG units for the same Bus of Figure 7.

For verifying the performance of NMCA, eight other methods (Firefly algorithm (FA), Grey Wolf Optimizer (GWO), particle swarm optimization (PSO), Improved invasive weed optimization algorithm (IWO), Artificial Bee Colony (ABC) Optimization, Harmony Search (HS), modified Camel Algorithm MCA and Salp Swarm Algorithm (SSA)) are applied on the IEEE 33 and IEEE 69 systems (in case of normal and heavy load). For each algorithm in case (1) has been 20 trials, and the other cases have been 30 trials (in MATLAB program), and for 
each run, 400 iterations with 400 particles. The results of these cases are shown in tables (1) to table (4). From Tables (1-4). The following points can be concluded:

1. Minimum cost and minimum mean cost (in all cases) find by The NMCA algorithm.

2. The performance of NMCA is significantly better than that of MCA, in all cases, wherein case (4) for mean cost improved (14.72\%).

3. The numerical results have verified the effectiveness of NMCA on the eight other methods.

Table 1. Eight methods outcomes for the IEEE 33-bus system (normal load).

\begin{tabular}{lccl}
\hline $\begin{array}{l}\text { optimization } \\
\text { Algorithms }\end{array}$ & Min cost & Max cost & Mean cost \\
\hline ABC & 3.297414 & 3.337504 & 3.317941 \\
MCA & 3.292848 & 3.394979 & 3.324087 \\
FA & 3.282356 & 3.406839 & 3.323749 \\
GWO & 3.282579 & 3.634918 & 3.323069 \\
HS & 3.297804 & 3.347978 & 3.321397 \\
IWO & 3.293783 & 3.382496 & 3.31921 \\
PSO & 3.290107 & 3.345048 & 3.31598 \\
SSA & 3.287941 & 3.634899 & 3.362063 \\
NMCA & 3.282302 & 3.316998 & 3.300953 \\
\hline
\end{tabular}

Table 2. Eight methods outcomes for IEEE 33-bus system (heavy load). optimization

\begin{tabular}{llll} 
Algorithms & Min cost & Max cost & Mean cost \\
\hline ABC & 19.75975 & 21.8354 & 20.40861 \\
MCA & 19.70274 & 23.54049 & 21.05797 \\
FA & 19.52142 & 19.77067 & 19.58841 \\
GWO & 19.51542 & 19.72042 & 19.56593 \\
HS & 19.51121 & 19.64605 & 19.58954 \\
IWO & 19.52976 & 22.9247 & 20.25597 \\
PSO & 19.51977 & 19.80548 & 19.62532 \\
SSA & 19.51679 & 20.11197 & 19.71944 \\
NMCA & 19.5 & 19.683 & 19.56436 \\
\hline
\end{tabular}

Table 3. Eight methods outcomes for the IEEE 69-bus system (normal load).

\begin{tabular}{llll}
\hline $\begin{array}{l}\text { optimization } \\
\text { Algorithms }\end{array}$ & Min cost & Max cost & Mean cost \\
\hline ABC & 2.868379 & 3.033751 & 2.943347 \\
MCA & 2.851789 & 3.251348 & 2.965185 \\
FA & 2.851863 & 4.125542 & 3.129453 \\
GWO & 2.851981 & 3.312213 & 2.97743 \\
HS & 2.87181 & 3.143175 & 3.039544 \\
IWO & 2.851788 & 3.376668 & 3.084053 \\
PSO & 2.908849 & 3.275703 & 3.030924 \\
SSA & 2.85179 & 3.402151 & 3.106977 \\
NMCA & 2.851787 & 3.054887 & 2.858705 \\
\hline
\end{tabular}

Table 4. Eight methods outcomes for IEEE 69-bus system (heavy load).

\begin{tabular}{lccc}
\hline $\begin{array}{l}\text { optimization } \\
\text { Algorithms }\end{array}$ & Min cost & Max cost & Mean cost \\
\hline ABC & 11.93381 & 12.26654 & 12.14651 \\
MCA & 12.09206 & 16.05889 & 13.79611 \\
FA & 11.74758 & 13.41285 & 12.25348 \\
\hline
\end{tabular}




\begin{tabular}{llll}
\hline GWO & 11.74021 & 12.12139 & 11.9055 \\
HS & 11.88661 & 12.12976 & 11.99741 \\
IWO & 11.73223 & 15.15766 & 12.56227 \\
PSO & 11.73144 & 12.59769 & 11.80033 \\
SSA & 11.74168 & 12.86283 & 12.22365 \\
NMCA & 11.70647 & 12.09886 & 11.76586 \\
\hline
\end{tabular}

The minimum cost and minimum mean cost in two cases normal and heavy load for 69-bus system reached by NMCA algorithms. Implement the NMCA algorithm to discover the best PVDG unit allocation to reduce traffic congestion, reduce power loss, and keep all bus voltages within an acceptable range. The NMCA algorithm is run on two study systems: an IEEE 33-bus system and an IEEE 69-bus system.

\section{Discussion on NMCA convergence}

The convergence rate $(\mathrm{CR})$ value is determined for all the investigated situations to test the convergence speed to further examine the performance of the proposed NMCA to handle the primary problems in the distribution grid (power losses, congestion, and voltage profile) as follows [15]:

$C R=\left(1-\frac{N F E^{R}}{N F E^{\max }}\right) \times 100 \%$

$N F E$ : evaluations number.

$N F E^{\max }$ : the max of NFE.

The worst and best convergence profiles of the considered method are indicated by $\mathrm{CR} \in[0,1]$, and the values of CR equal to zero and unity, respectively. [15] is the adopted technique for determining the value of CR for any number of optimization methods:

1. Run all the optimization methods up to $\mathrm{NFE}^{\max }$

2. The value of interest is determined as the defined minimum objective value (in the case of the minimization issue).

3. Determine which NFE each algorithm has reached this value and mark it as NFER in that NFE.

4. Calculate the $\mathrm{CR}$ for each algorithm using the formula indicated in equation (25).

The best-obtained convergence profiles of the observed test cases are used to calculate the CR value. Table 5 shows that the suggested NMCA algorithm has the greatest average CR value, indicating that it converges to the optimum solution in a less number of NFFEs than any of the other approaches considered. Figures 9 and 10 show comparative graphs of the fitness function for all of the optimization approaches investigated. In most circumstances, NMCA finds the lowest fitness value with a faster convergence profile. In this investigation, it outperforms the other examined algorithms.

Table 5. The values of CR running over many methods.

\begin{tabular}{lll}
\hline \multirow{2}{*}{ Algorithms } & \multicolumn{2}{c}{ CR\% } \\
\cline { 2 - 3 } & IEEE 33 bus & IEEE 69 bus \\
\hline
\end{tabular}




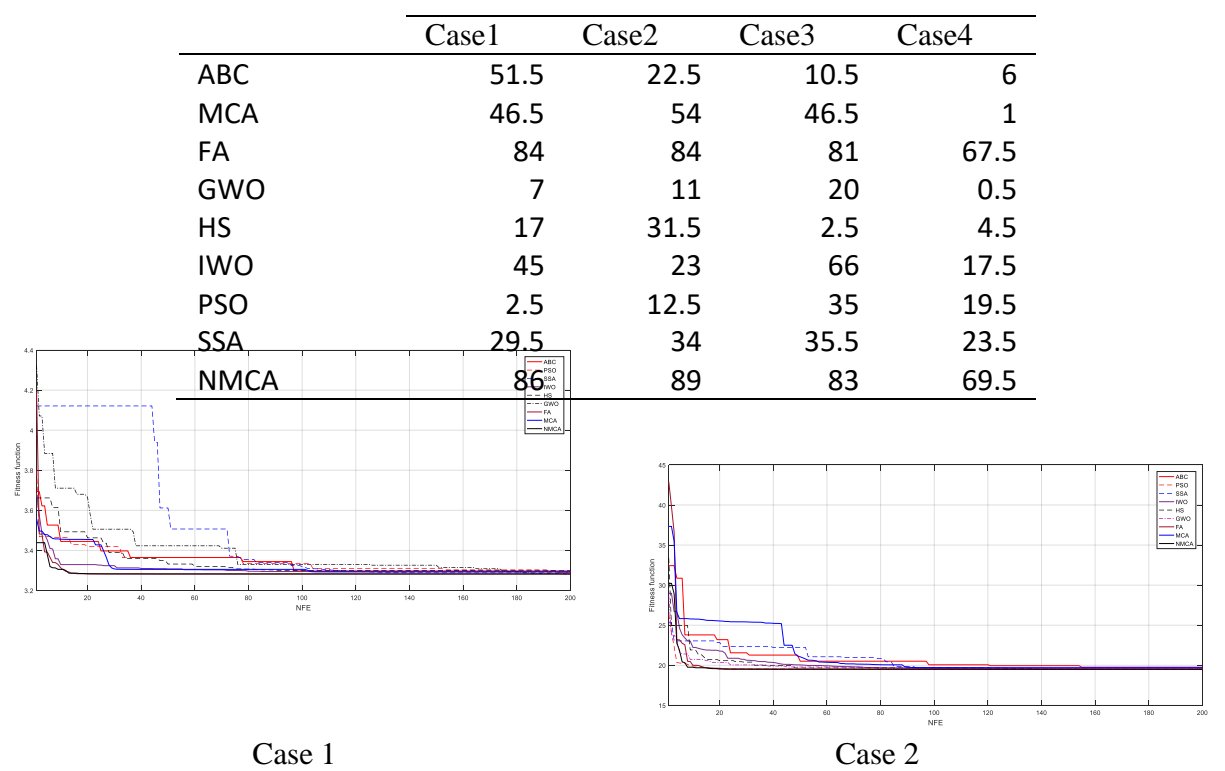

Fig. 9. Fitness function convergence over various algorithms over IEEE 33-bus: Case 1 and Case2

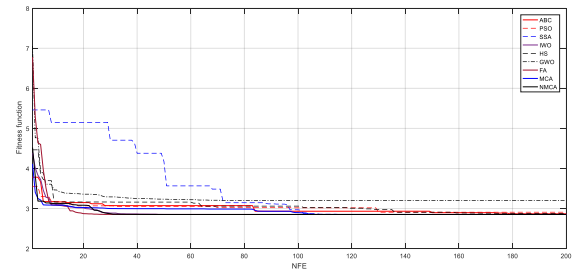

Case 3

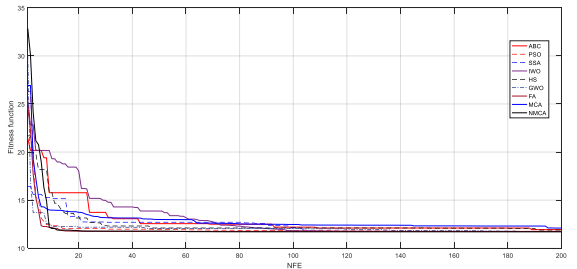

Case 4

Fig. 10. Fitness function convergence over various algorithms about IEEE 69-bus: Case 3 and Case 4

\section{Conclusion}

An optimal configuration of PVDGs to relieve congestion problems in the power system has been adopted in this work. This was accomplished using a unique NMCA algorithm. The NMCA was inspired by camels' behaviour in searching for food and water in the desert. The suggested approach is fairly basic, and it only needs a few parameters to modify, therefore it can be used to a broader class of optimization issues than just engineering problems. The validity of the NMCA is tested utilising power system issues. The overloaded lines, voltage profile, and power losses in the distribution system are all taken into account. On IEEE 33-bus and IEEE 69-bus test power systems, the suggested NMCA approach has been effectively implemented. The present method can outperform the well-known algorithms (like ABC, MCA, FA, GWO, HS, IWO, PSO, and SSA) and the convergence of the algorithm is evaluated here using convergence rate and convergence plots. The suggested technique is also very effective for limited engineering situations, according to the results. Because NMCA is so effective, it's possible to make enhancements in design, such as for multi-objective and high-dimensional 
optimization problems. Future researchers will find NMCA to be an effective optimization technique for dealing with multi-objective large-scale power system challenges.

\section{References}

[1] Pillai, D.S.; Rajasekar, N. A comprehensive review on protection challenges and fault diagnosis in PV systems. Renewable \& sustainable energy reviews 2018, 91, 18-40.

[2] Duong, M.Q.; Pham, T.D.; Nguyen, T.T.; Doan, A.T.; Tran, H.V. Determination of Optimal Location and Sizing of Solar Photovoltaic Distribution Generation Units in Radial Distribution Systems. Energies 2019, 12, 174.

[3] Sahab, M.G.; Toropov, V.V.; Gandomi, A.H. 2 - A Review on Traditional and Modern Structural Optimization: Problems and Techniques. In Metaheuristic Applications in Structures and Infrastructures, Gandomi, A.H., Yang, X.-S., Talatahari, S., Alavi, A.H., Eds.; Elsevier: Oxford, 2013; pp. 25-47.

[4] Rao, R.S.; Ravindra, K.; Satish, K.; Narasimham, S.V.L. Power Loss Minimization in Distribution System Using Network Reconfiguration in the Presence of Distributed Generation. IEEE Transactions on Power Systems 2013, 28, 317-325, doi:10.1109/TPWRS.2012.2197227.

[5] Heidari, M.A. Optimal network reconfiguration in distribution system for loss reduction and voltageprofile improvement using hybrid algorithm of PSO and ACO. CIRED - Open Access Proceedings Journal 2017, 2017, 2458-2461.

[6] Ali, R.S.; Alnahwi, F.M.; Abdullah, A.S. A modified camel travelling behaviour algorithm for engineering applications. Australian Journal of Electrical and Electronics Engineering 2019, 16, 176-186, doi:10.1080/1448837X.2019.1640010.

[7] Yang, X.-S. Nature-Inspired Optimization Algorithms: Second Edition; 2020.

[8] Mohammed Khalid, I.; Ramzy Salim, A. Novel Optimization Algorithm Inspired by Camel Traveling Behavior. Iraqi Journal for Electrical And Electronic Engineering 2016, 12, 167-177.

[9] Abdul Kadir, A.F.; Mohamed, A.; Shareef, H.; Wanik, M.Z.C.; Ibrahim, A.A. Optimal sizing and placement of distributed generation in distribution system considering losses and THDv using gravitational search algorithm. Prz. Elektrotech. Przeglad Elektrotechniczny 2013, 89, 132-136.

[10] Stefopoulos, G.K.; Fang, Y.; Cokkinides, G.J.; Meliopoulos, A.P.S.; Proceedings. 37th North American Power, S. Advanced contingency selection methodology. 2005, 67-73.

[11] Mukherjee, V.; Verma, S. Optimal real power rescheduling of generators for congestion management using a novel ant lion optimizer. IET Generation, Transmission \& Distribution 2016, 10, doi:10.1049/iet-gtd.2015.1555.

[12] Doyle, J.C. Feedback Control Theory. 2013.

[13] Wazir, A.; Arbab, N. Analysis and Optimization of IEEE 33 Bus Radial Distributed System Using Optimization Algorithm. 2016.

[14] Gonzalez, G.; Barragan, L.; Rivas, E. Locating distributed generation units in radial systems. Contemporary Engineering Sciences 2017, 10, 1035-1046, doi:10.12988/ces.2017.79112.

[15] Aman, M.M.; Jasmon, G.B.; Bakar, A.H.A.; Mokhlis, H. A new approach for optimum simultaneous multi-DG distributed generation Units placement and sizing based on maximization of system loadability using HPSO (hybrid particle swarm optimization) algorithm. Energy 2014, 66, 202-215, doi:https://doi.org/10.1016/j.energy.2013.12.037.

[16] Gandomi, A.H. Interior search algorithm (ISA): A novel approach for global optimization. ISA Transactions 2014, 53, 1168-1183, doi:https://doi.org/10.1016/j.isatra.2014.03.018. 